\title{
Diurnal Variation of Blood Pressure in Patients with Salt Sensitive Hypertension
}

\author{
Hiroaki Matsuoka*, Kazushige Fukui, Hiroshi Hayakawa, Etsu Suzuki, Akihiro Tojo, \\ Shinichiro Namba, Keiichiro Atarashi, Yasunobu Hirata,and Tsuneaki Sugimoto
}

\begin{abstract}
Variations of blood pressure (BP) and heart rate (HR) were evaluated in patients with essential hypertension (EH) under the conditions of low salt and high salt intake. Fourteen patients with EH were hospitalized and consecutively kept on a daily salt intake of 3 and $20 \mathrm{~g}$ for 1 week each. Variations in BP were measured at intervals of 15 or 30 min using automatic noninvasive BP recorders. Urinary excretion of norepinephrine (UNEV) was also measured serially. The patients whose mean BP was increased more than $10 \%$ by salt loading were classified as salt sensitive hypertensives (SS; $n=6$ ), while the rest were defined as salt resistant hypertensives $(\mathrm{SR} ; n=8)$. In SR, averages of both systolic and diastolic blood pressure at night were significantly lower than those during the daytime for both low salt and high salt conditions. UNEV in SR was also decreased significantly at night in both conditions. In SS, however, the nocturnal fall in blood pressure was not significant during salt loading. Furthermore, in SS, UNEV was not decreased significantly during the night in either condition. These results suggest that salt loading in addition to incomplete inhibition of the sympathetic nervous system at night may be involved in the absence of a nocturnal decrease in BP in patients with salt sensitive hypertension.(Hypertens Res 1993; 16: 233-237)
\end{abstract}

Key Words: salt sensitive hypertension, blood pressure variation, sympathetic nervous system

Blood pressure shows physiological diurnal variations with higher levels usually occurring during the day and lower levels during the night. Although the controlling mechanisms of diurnal variations in blood pressure remain uncertain, it is likely that several factors are involved. These factors include the sympathetic nervous system $(1,2)$, the reninangiotensin-aldosterone system $(3,4)$, and the pituitary adrenal system (5). It is well known that the sympathetic nervous system and sodium ion play important roles in blood pressure regulation. In the present study we examined the relationship between blood pressure variation and various endogenous humoral factors and sympathetic nervous activity in patients with salt sensitive and salt resistant hypertension under the conditions of low and high salt intake.

\section{Subjects and Methods}

The study comprised 14 patients with essential hypertension, 9 men and 5 women, aged from 39 to 63 years ( $51 \pm 3\langle\mathrm{SE}\rangle$ years). They were all hospitalized and kept on a daily sodium intake of $8 \mathrm{~g}$; and all medications were withheld for at least two weeks prior to the study. Diagnosis was based on history, physical findings, .urinalysis, biochemical and endocrinological examinations of blood samples and radiological studies. All patients had normal renal function and were assessed to be in hypertensive stage I or II according to the WHO classification. Informed consent was received from all patients. A low sodium diet $(\mathrm{NaCl} 3 \mathrm{~g} /$ day) was given for a week, followed by a high sodium diet $(\mathrm{NaCl} 20$ $\mathrm{g}$ /day) for a week. On the last day of each period, blood samples were taken at $0800 \mathrm{~h}$ from the patients who had been fasting overnight and who were at rest in the supine position. 24-h urine was collected separately during the daytime $(0600 \mathrm{~h}$ to 2100 h) and at night $(2100 \mathrm{~h}$ to $0600 \mathrm{~h})$. Plasma renin activity (PRA) and plasma concentrations of aldosterone (PAC) were measured by radioimmunoassay. Atrial natriuretic peptide (PANP) was measured by radioimmunoassay as previously described (6). Plasma norepinephrine concentration (PNE) and urinary excretion of norepinephrine (UNEV) were determined by high performance liquid chromatography as described previously (7). 24-h ambulatory blood pressure and heart rate were monitored every $15 \mathrm{~min}$ during the daytime $(0600 \mathrm{~h}$

From the Second Department of Internal Medicine, University of Tokyo, Tokyo, * Division of Hypertension \& Renal Diseases, National Cardiovascular Center, Suita, Osaka, Japan.

This study was supported in part by Research Grant 63-6 for Cardiovascular Diseases from the Ministry of Health and Welfare, Japan.

Address for Reprints: Hiroaki Matsuoka, M.D.,Division of Hypertension \& Renal Diseases, National Cardiovascular Center, 5-7-1, Fujishiro-dai, Suita, Osaka 565, Japan.

Received November 10,1992; accepted in revised form August 5,1993. 
to $2100 \mathrm{~h})$ and every $30 \mathrm{~min}$ at night $(2100 \mathrm{~h}$ to $0600 \mathrm{~h}$ ) using a noninvasive, automatic sphygmomanometer (ABPM 630; Nippon Colin, Komaki, Japan). This device measured blood pressure by the Korotkoff as well as the oscillometric method. In the present study the value of blood pressure was expressed by the oscillometric method. All patients went to bed at $2100 \mathrm{~h}$ and woke up at $0600 \mathrm{~h}$. By filling out a questionnaire we decided whether patients had slept well or not. Patients who complained of sleep disturbance on the day of blood pressure monitoring were excluded from the study. Systolic and diastolic blood pressures (SBP and DBP) and heart rate (HR) were averaged over 60min intervals. Average SBP, DBP and HR for the waking $(0800 \mathrm{~h}$ to $2100 \mathrm{~h})$ and sleeping periods $(0000 \mathrm{~h}$ to $0400 \mathrm{~h})$ were determined. Blood pressure monitoring was performed in a ward and the patients were permitted to carry out their usual activities in the ward.

\section{Statistical Analysis}

Averages of SBP, DBP and HR during waking hours $(0800 \mathrm{~h}$ to $2100 \mathrm{~h})$ were compared with nighttime $(0000 \mathrm{~h}$ to $0400 \mathrm{~h})$ values using one-way analysis of variance and Student's $t$-test. Linear regression analysis was conducted on blood pressure and heart rate. 1-h blood pressure and heart rate variabilities were evaluated by coefficients of variation $(\mathrm{CV}$; standard deviation/mean of $24 \mathrm{~h} \times 100)$. Data are presented as mean \pm standard error. Student's unpaired $t$-test was used for group comparison and paired $t$-test was used for low salt and high salt comparison. A $p$ value less than 0.05 was considered significant.

\section{Results}

The patients were divided into two groups. The patients whose 24-h averaged MBP was increased more than 10 percent by sodium loading were classified as salt sensitive hypertensives (SS; $n=6,3$ men, 3 women, mean age $53 \pm 4$ years), while the rest were defined as salt resistant hypertensives (SR; $n=8,6$ men, 2 women, mean age $50 \pm 3$ years). The average increase in blood pressure induced by salt loading was $23 / 10 \mathrm{mmHg}$ in SS group and $9 / 5 \mathrm{mmHg}$ in SR group.

Variations in Blood Pressure and Heart Rate in Salt Sensitive and Resistant Hypertensives

24-h averaged SBP, DBP, mean blood pressure $\{(\mathrm{SBP}-\mathrm{DBP}) / 3+\mathrm{DBP} ; \mathrm{MBP}\}$ and HR were similar in both groups during the low salt period. HR was significantly decreased at night in both groups during the low salt and high salt periods. SBP and DBP at night were significantly lower than those in the day in the SR group during both periods (Fig. 1 upper panel). In the SS group, nocturnal falls in SBP and MBP were not significant during low or high salt intake, and the significant nocturnal fall in DBP during the low salt period was eliminated by salt loading (Fig. 1 lower panel).
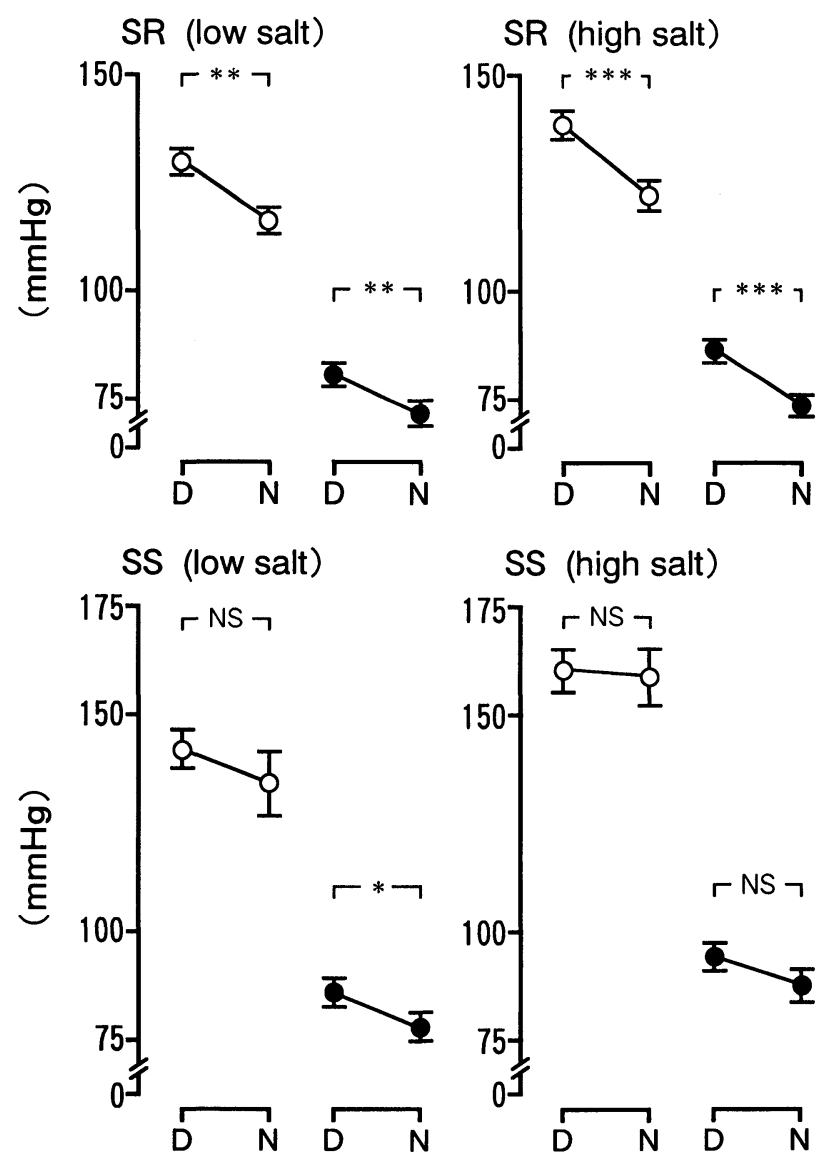

Fig. 1. $S B P$ and DBP in the daytime and at night during the low salt and high salt periods in the SR group (upper panel) and the SS group (lower panel). $\bigcirc, S B P$;,$D B P$; $D$, daytime; $N$, nighttime; ${ }^{*} \mathrm{p}<0.05 ;{ }^{* *} \mathrm{p}<0.01 ;{ }^{* * *} \mathrm{p}<$ $0.001 ; N S$, not significant.

During low salt intake, the variation in HR was parallel to that of SBP and DBP in both groups. However, during high salt intake, there was no significant relationship between HR and SBP in the SS group $(r=0.20)$, although a significant positive relationship was observed in the SR group $(r=0.68, p$ $<0.001)$. The CV values of SBP, DBP, MBP, and HR were similar in both groups and were not affected by changes of salt balance.

Renal Function and Humoral Factors during Low Salt and High Salt Intake in Salt Sensitive and Resistant Hypertensives

Creatinine clearance and urinary sodium excretion (UNaV), and values of PRA, PAC, PANP, PNE, and UNEV are presented in Table 1 . There was no significant difference in creatinine clearance between the two groups. PRA in SS during low salt intake was significantly lower than that in SR and was significantly suppressed by salt loading in both groups (in SS, $3.1 \pm 0.9 \mathrm{ng} / \mathrm{ml} / \mathrm{h}$ to $1.5 \pm 0.3, p<$ 0.5 ; in SR, $6.0 \pm 0.8$ to $1.8 \pm 0.6, p<0.01)$. PAC was significantly inhibited by salt loading in both groups; however, there was no difference in PAC 
Table 1. Renal Function, Urinary Sodium Excretion (UNaV), and Humoral Factors during Low Salt and High Salt Periods in Salt Sensitive and Salt Resistant Hypertensives

\begin{tabular}{lcccc}
\hline & \multicolumn{2}{c}{ Salt Sensitive Group } & \multicolumn{2}{c}{ Salt Resistant Group } \\
& Low Salt & High Salt & Low Salt & High Salt \\
\hline Ccr $(\mathrm{ml} / \mathrm{min})$ & $110 \pm 12$ & & $112 \pm 9$ & \\
UNaV (mEq/day) & $38 \pm 5$ & $224 \pm 11^{\mathrm{c}}$ & $32 \pm 3$ & $223 \pm 11^{\mathrm{c}}$ \\
PRA $(\mathrm{ng} / \mathrm{ml} / \mathrm{h})$ & $3.1 \pm 0.9^{\mathrm{a}}$ & $1.5 \pm 0.3^{\mathrm{b}}$ & $6.0 \pm 0.8$ & $1.8 \pm 0.6^{\mathrm{c}}$ \\
PAC $(\mathrm{ng} / \mathrm{dl})$ & $21.2 \pm 3.8$ & $12.4 \pm 2.5^{\mathrm{c}}$ & $19.0 \pm 2.0$ & $7.5 \pm 0.9^{\mathrm{c}}$ \\
PANP $(\mathrm{pg} / \mathrm{ml})$ & $40 \pm 7$ & $65 \pm 13^{\mathrm{b}}$ & $43 \pm 4$ & $77 \pm 14^{\mathrm{b}}$ \\
PNE $(\mathrm{pg} / \mathrm{ml})$ & $341 \pm 134$ & $178 \pm 81^{\mathrm{b}}$ & $242 \pm 61$ & $205 \pm 42^{\mathrm{b}}$ \\
UNEV $(\mu \mathrm{g} / \mathrm{day})$ & $34.1 \pm 4.7$ & $23.1 \pm 3.0^{\mathrm{b}}$ & $31.1 \pm 6.0$ & $19.8 \pm 3.9^{\mathrm{b}}$ \\
\hline
\end{tabular}

Ccr: creatinine clearance. ${ }^{\mathrm{a}} p<0.05$ vs. salt resistant, ${ }^{\mathrm{b}} p<0.05$ vs. low salt, ${ }^{\mathrm{c}} p<0.01 v s$. low salt.

between the two groups. PANP in SS was similar to that in SR during low salt intake and was increased by salt loading in both groups. There was no significant difference in PNE between SS and SR, and salt loading significantly decreased PNE in the two groups. There was no difference in whole-day UNEV between the two groups and salt loading significantly decreased whole-day UNEV in both groups. UNEV at nighttime was significantly lower than that at daytime in SR during both the low and high salt periods $(1.05 \pm 0.25 \mu \mathrm{g} / \mathrm{h} v s .1 .51 \pm 0.25$, $p<0.001$ in the low salt period and $0.70 \pm 0.16 v s$. $0.92 \pm 0.17, p<0.02$ in the high salt period; Fig. 2 left panel). However, in the SS group there was no significant difference in UNEV between the nighttime and daytime $(1.26 \pm 0.17 v s .1 .55 \pm 0.23$ in the low salt period and $0.80 \pm 0.09$ vs. $1.08 \pm 0.18$ in the high salt period; Fig. 2 right panel).

\section{Discussion}

It is well recognized that blood pressure follows a diurnal variation, increasing during the day (during waking hours) and falling at night (during sleeping hours). HR exhibits a similar pattern in normoten- sive subjects and patients with essential hypertension $(8,9)$. The precise mechanisms for the nocturnal fall in blood pressure are unknown; however, suppression of sympathetic nervous activity may be deeply involved $(10,11)$. A reduction in sympathetic nervous activity during sleep may lead to a reduction in blood pressure at night. It has been reported that plasma norepinephrine concentration and urinary norepinephrine excretion are elevated during the daytime and reduced during sleep $(12,13)$.

In the present study patients with essential hypertension were divided into two groups according to their sodium sensitivities $(14,15)$. Six out of 14 patients with essential hypertension were classified as salt sensitive hypertensives. There were no differences with respect to mean age and renal function between the SS and SR groups. PRA in SS was significantly lower than that in SR during low salt intake. PAC, PANP, PNE and whole day UNEV in SS were similar to those in SR.

In our study, a significant nocturnal fall in blood pressure was observed in the SR group. However, there was no significant nocturnal fall in blood pressure in patients with salt sensitive hypertension during high salt intake. The renin angiotensin sys-

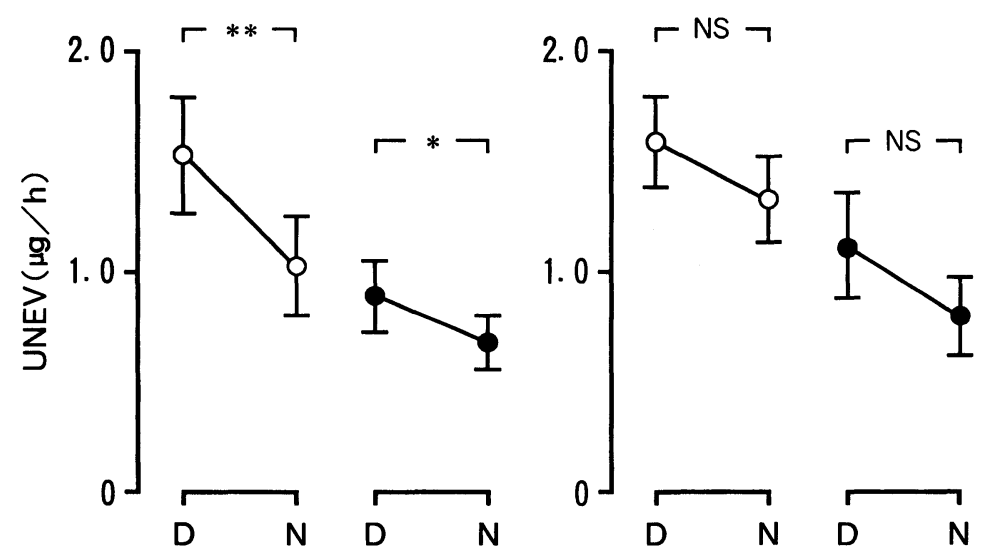

Fig. 2. UNEV (urinary norepinephrine excretion) in the daytime and at night during the low salt and high salt periods in the SR group (left panel) and the SS group (right panel). $\bigcirc$, low salt period; $\bigcirc$, high salt period; D, daytime; N, nighttime; ${ }^{*} \mathrm{p}<0.02 ;{ }^{* *} \mathrm{p}<0.001 ; N S$, not significant. 
tem, circulating and urinary catecholamines, the pituitary adrenal axis, plasma volume and the autonomic nervous system may be involved in blood pressure variations, because an absence of a nocturnal fall in blood pressure has been reported in several disease states, such as malignant hypertension (16), pheochromocytoma (17), Cushing syndrome (5), autonomic nervous failure (18), and preeclampsia (19), although controversial results have been reported in patients with pheochromocytoma (20). In the present study there were no significant differences in circulating humoral factors between the SS and SR groups, except that PRA in the SS group during the low salt diet was significantly lower than that in the SR group. Although plasma volume was not measured in our study, a low PRA might reflect plasma volume expansion. If this is the case, it is possible that expanded plasma volume may affect blood pressure variation, since different blood pressure rhythms have been reported in patients with primary aldosteronism (4).

With regard to the sympathetic nervous system, 24-h UNEV was not significantly different between the two groups and salt loading decreased it significantly in both groups. However, in the SS group UNEV was not decreased significantly at night irrespective of the changes in sodium balance, although a significant reduction in UNEV was observed at night in the SR group during both periods. Fujita et al. (15) have reported that relatively higher activity of the sympathetic nervous system may contribute to an elevation of blood pressure during sodium loading in salt sensitive hypertensive patients. These results suggest that salt loading, in addition to the incomplete inhibition of the sympathetic nervous system at night, may be involved in the absence of blood pressure reduction at night in patients with salt sensitive hypertension. The mechanisms for the incomplete suppression of sympathetic nervous activities remain to be elucidated.

The diurnal variation of blood pressure is well correlated with that of HR in patients with salt resistant hypertension. In salt sensitive hypertensives, the positive relationship between SBP and HR disappeared during high salt diet. However, the nocturnal fall in HR was retained even in patients with salt sensitive hypertension during high salt intake. Many factors are presumed to be involved in blood pressure variation, while HR is controlled mainly by the autonomic nervous system (i.e. sympathetic and parasympathetic activities $(21,22))$. Guazzi et al. $(23)$ and Zemaityte et al. (24) have reported that sleep bradycardia is due to an increase in parasympathetic nervous activity. Thus, the nocturnal fall in HR may be mainly due to increased parasympathetic nervous activity, though inhibition of sympathetic nervous activity is thought to play some role. Therefore, it is suggested that parasympathetic nervous activity remains unchanged at night during high salt intake and that the significant positive relationship between SBP and HR disappears in patients with salt sensitive hypertension.

In conclusion, salt loading under the condition of incomplete inhibition of sympathetic nervous activity at night may contribute to the absence of a nocturnal fall in blood pressure in patients with salt sensitive hypertension.

\section{References}

1. Tuck ML, Stern N, Sowers JR: Enhanced 24-hour norepinephrine and renin secretion in young patients with essential hypertension: Relation with the circadian pattern of arterial blood pressure. Am J Cardiol 1985; 55: 112-115.

2. Sowers JR: Dopaminergic control of circadian norepinephrine levels in patients with essential hypertension. J Clin Endocrinol Metab 1981; 53: 1133-1137.

3. Tsuchiya $\mathbf{M}$, Kojima $\mathrm{S}$, Nakagawa $\mathbf{M}$, et al: Home blood pressure and circadian variation of blood pressure in the evaluation of hypertensive patients. Jpn Circ J 1981; 45: 772-780.

4. Tanaka T, Natsume T, Shibata $\mathrm{H}$, et al: Circadian rhythm of blood pressure in primary aldosteronism and renovascular hypertension-Analysis by the cosinor method-. Jpn Circ J 1983; 47: 788-794.

5. Imai Y, Abe K, Sasaki S, et al: Altered circadian blood pressure rhythm in patients with Cushing's syndrome. Hypertension 1988; 12: 11-19.

6. Hirata $\mathrm{Y}$, Ishii $\mathrm{M}$, Matsuoka $\mathrm{H}$, et al: Plasma concentrations of atrial natriuretic peptide and cyclic GMP in patients with heart diseases. Am Heart J 1987; 113: 1463-1469.

7. Ishii $\mathrm{M}$, Ikeda $\mathrm{T}$, Takagi $\mathrm{M}$, et al: Elevated plasma catecholamines in hypertensives with primary glomerular diseases. Hypertension 1983; 5: 545-551.

8. Mancia G, Ferrari A, Gregorini L, et al: Blood pressure and heart rate variabilities in normotensive and hypertensive human beings. Circ Res 1983; 53: 96-104.

9. Tochikubo O, Miyazaki N, Kaneko Y: Relationship between 24-hour arterial pressure and heart rate variation in normotensives, hypertensives and patients with Shy-Drager syndrome. Jpn Circ J 1987; 51: 485-494.

10. Akerstedt T, Levi L: Circadian rhythms in the secretion of cortisol, adrenaline and noradrenaline. Eur $J$ Clin Invest 1978; 8: 57-58.

11. Stene M, Panagiotis N, Tuck ML, Sowers JR, Mayes D, Berg G: Plasma norepinephrine levels are influenced by sodium intake, glucocorticoid administration, and circadian changes in normal man. J Clin Endocrinol Metab 1980; 51: 1340-1345.

12. Maling TJB, Dollery CT, Hamilton CA: Clonidine and sympathetic activity during sleep. Clin Sci 1979; 57: $509-514$.

13. Townshend MM, Smith AJ: Factors influencing the urinary excretion of free catecholamines in man. Clin Sci 1973; 44: 253-265.

14. Kawasaki T, Delea CS, Bartter FC, Smith H: The effect of high-sodium and low-sodium intakes on blood pressure and other related variables in human subjects with idiopathic hypertension. Am J Med 1978; 64: 193-198.

15. Fujita T, Henry WL, Bartter FC, Lake CR, Delea $\mathrm{CS}$ : Factors influencing blood pressure in salt-sensitive patients with hypertension. Am J Med 1980; 69: 334-344.

16. Shaw DB, Knapp MS, Davies DH: Variations of blood-pressure in hypertensives during sleep. Lancet 1963; i: 797-799.

17. Littler WA, Honour AJ: Direct arterial pressure, heart rate, and electrocardiogram in unrestricted pa- 
tients before and after removal of a phaeochromocytoma. Quart J Med 1974; 43: 441-449.

18. Mann S, Altman DG, Raftery EB, Bannister R: Circadian variation of blood pressure in autonomic failure. Circulation 1983; 68: 477-483.

19. Redman CWG, Beilin LJ, Bonnar J: Reversed diurnal blood pressure rhythm in hypertensive pregnancies. Clin Sci Mol Med 1976; 51: 687s-689s.

20. Imai Y, Abe K, Miura Y, et al: Hypertensive episodes and circadian fluctuations of blood pressure in patients with phaeochromocytoma: studies by longterm blood pressure monitoring based on a volumeoscillometric method. J Hypertens 1988; 6: 9-15.

21. Levy MN, Zieske H: Autonomic control of cardiac pacemaker activity and atrioventricular transmission. J Appl Physiol 1969; 27: 465-470.

22. Vatner SF, Higgins CB, Braunwald E: Sympathetic and parasympathetic components of reflex tachycardia induced by hypotension in conscious dogs with and without heart failure. Cardiovasc Res 1974; 8: 153-161.

23. Guazzi M, Mancia G, Kumazawa T, Baccelli G, Zanchetti A: Effects of cardiac denervation on blood pressure and heart rate during natural sleep in the cat. Cardiovasc Res 1968; 3: 265-270.

24. Zemaityte D, Varoneckas G, Sokolov E: Heart rhythm control during sleep. Psychophysiology 1984; 21: $279-288$. 